\title{
Uncharted Frontiers in the Spectroscopy of Highly Charged lons
}

P. Beiersdorfer, G. Brown, J. Crespo, S-H. Kim, P. Neill, E. Träbert, J. Tschischgale, S. Utter, K. Widmann

January 8, 2000

U.S. Department of Energy

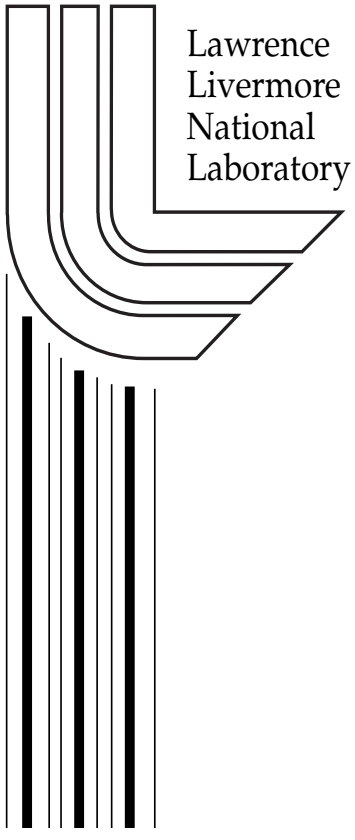




\section{DISCLAIMER}

This document was prepared as an account of work sponsored by an agency of the United States Government. Neither the United States Government nor the University of California nor any of their employees, makes any warranty, express or implied, or assumes any legal liability or responsibility for the accuracy, completeness, or usefulness of any information, apparatus, product, or process disclosed, or represents that its use would not infringe privately owned rights. Reference herein to any specific commercial product, process, or service by trade name, trademark, manufacturer, or otherwise, does not necessarily constitute or imply its endorsement, recommendation, or favoring by the United States Government or the University of California. The views and opinions of authors expressed herein do not necessarily state or reflect those of the United States Government or the University of California, and shall not be used for advertising or product endorsement purposes.

Work performed under the auspices of the U. S. Department of Energy by the University of California Lawrence Livermore National Laboratory under Contract W-7405-Eng-48.

This report has been reproduced directly from the best available copy.

Available to DOE and DOE contractors from the

Office of Scientific and Technical Information

P.O. Box 62, Oak Ridge, TN 37831

Prices available from (423) 576-8401

http://apollo.osti.gov/bridge/

Available to the public from the National Technical Information Service

U.S. Department of Commerce 5285 Port Royal Rd., Springfield, VA 22161 http://www.ntis.gov/

OR Lawrence Livermore National Laboratory Technical Information Department's Digital Library http://www.llnl.gov/tid/Library.html 


\title{
Uncharted Frontiers in the Spectroscopy of Highly Charged Ions
}

97-ERD-103

Final Report

Peter Beiersdorfer, Gregory Brown, José Crespo, Seong-Ho Kim, Paul Neill, Elmar Träbert, Jörg Tschischgale, Steven Utter, Klaus Widmann

\begin{abstract}
:
The development of novel techniques is critical for maintaining a state-of-the-art core competency in atomic physics and readiness for evolving programmatic needs. We have carried out a three-year effort to develop novel spectroscopic instrumentation that added new dimensions to our capabilities for measuring energy levels, radiative transition probabilities, and electron-ion excitation processes. The new capabilities created were in areas that heretofore had been inaccessible to scientific scrutiny and included high-resolution spectroscopy of hard $\mathrm{x}$ rays, femtosecond lifetime measurements, measurements of transition probabilities of long-lived metastable levels, polarization spectroscopy, ultra-precise determinations of energy levels, and the establishment of absolute wavelength standards in x-ray spectroscopy. Instrumentation developed during the period included a transmissiontype crystal spectrometer, a flat-field EUV spectrometer, and the development and deployment of absolutely calibrated monolithic crystals. The new capabilities enabled very sensitive tests of atomic wave functions, of calculations of magnetic sublevel populations, and of fundamental theories in uncharted regimes, and provided the basis for developing new diagnostic techniques of high-density plasmas.
\end{abstract}




\section{Introduction}

The recent availability of sources of highly charged ions has opened the possibility of studying atomic properties in unprecedented detail. Spectroscopic exploitation of these new resources, however, is by no measure complete or mature, and many areas of atomic physics are not accessible to scientific scrutiny. This project was intended to create innovative capabilities and to explore novel approaches to push back the frontiers of experimental atomic physics and to fully exploit unexplored opportunities. The capabilities created extended to all spectroscopic observables, i.e., photon energy, time dependence, and line intensity, and thus have broadened all aspects our spectroscopic capabilities.

In order to succeed in our objectives, we systematically created new research tools tailored to Livermore's novel ion sources that enable entirely new classes of measurements. We built new state-of-the-art instrumentation and developed novel techniques to work smarter. The new instrumentation includes a transmission-type crystal spectrometer for measuring high-energy x rays $(>20 \mathrm{keV})$ with high resolving power, a flat-field EUV spectrometer to access the 20-400 $\AA$ wavelength band, an absolutely calibrated crystal spectrometer based on monolithic crystals, a polarization-sensitive x-ray spectrometer, and an ultra-high resolution soft x-ray spectrometer.

Details of the new instrumentation and the scientific results obtained with each are given below. The results have been published in a large number of peer-reviewed publications. A list of these is given at the end.

\section{New Instrumentation Developed and Deployed under this LDRD Project}

\section{$\underline{\text { High-resolution hard x-ray spectrometer }}$}

Throughout the world, hard $\mathrm{x}$ rays with energies above about $15 \mathrm{keV}$ have been measured only with low-resolution solid-state type detectors. In order to address this deficiency and to extend LLNL's high-resolution spectroscopic capabilities to x rays above $15 \mathrm{keV}$, we developed a high-energy spectrometer based on transmission-type optics. Such spectrometers are widely used in nuclear physics, but they have not been used in atomic physics measurements where the count rates are extremely low.

Despite count rate limitations, we successfully implemented a transmission-type crystal spectrometer. Initial count rates were only as high as 1 count per hour. This was subsequently increased by factors of 10-20. The spectrometer's utility was demonstrated in spectral measurements of the heliumlike $\mathrm{Xe}^{52+} \mathrm{K}$-shell spectrum. All lines were clearly resolved in our spectrum validating the concept and its implementation. This is the very first time such high-energy lines $(>30 \mathrm{keV})$ have been resolved in a spectral measurement. We achieved a resolving power of 1200, which is far better than the resolving power of 80 achieved with high-purity germanium detectors that are usually used in atomic physics for 
the measurement of hard $x$ rays. With the new capability we have now opened up the hard $\mathrm{x}$-ray regime to experimental scrutiny. This is important given that NIF will be able to access such spectra and is expected to use them as diagnostics, as illustrated in Fig. 1. Indeed, the OMEGA laser has already achieved conditions where the K-shell spectrum of krypton can be produced putting that of xenon into close reach.

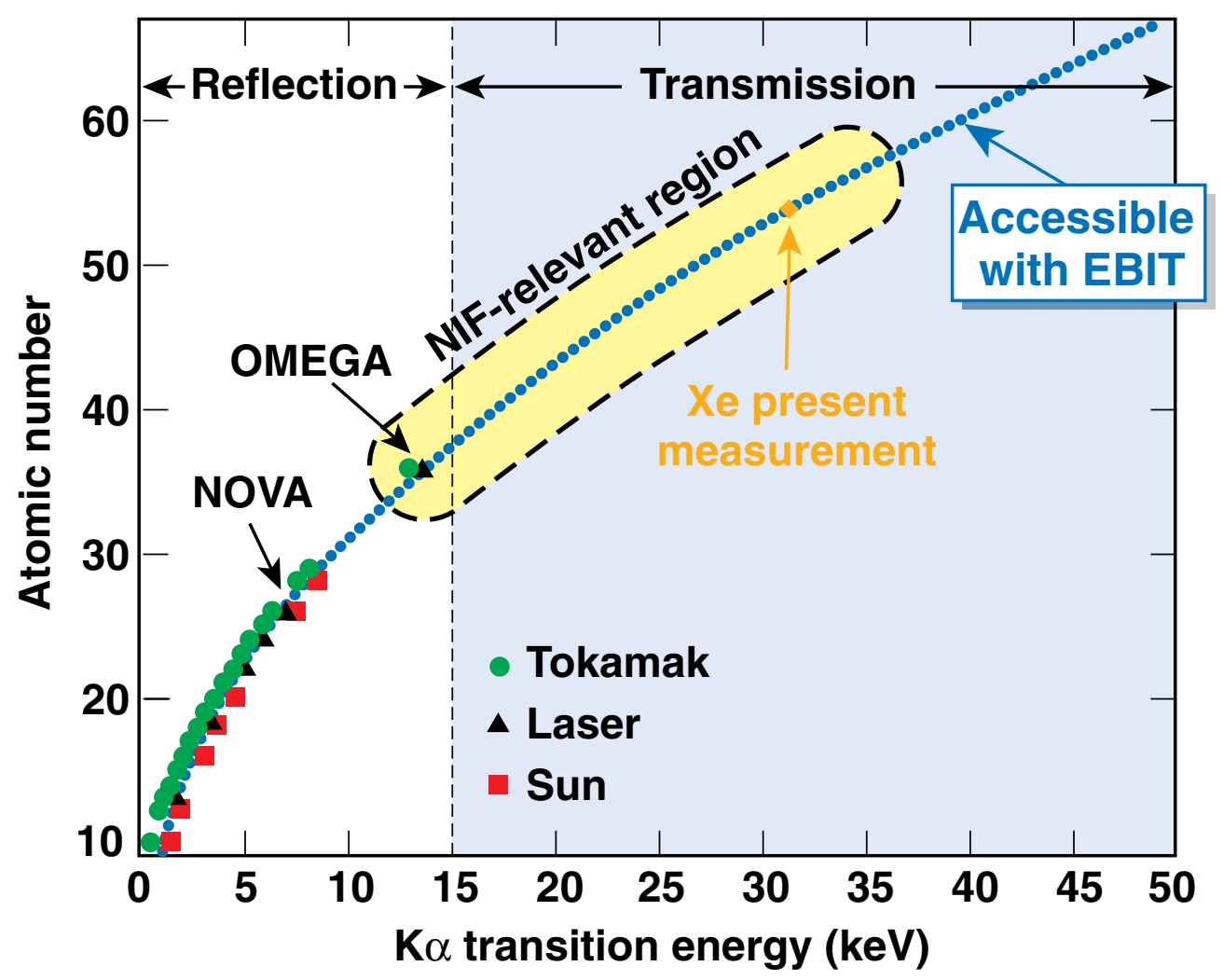

Fig. 1. Energy of the K-shell emission lines for different elements. Presentday plasma sources, such as tokamaks, the NOVA and Omega lasers, and our Sun, have produced $\mathrm{X}$-ray energies up to $15 \mathrm{keV}$ from elements with atomic number as high as krypton $(Z=36)$. NIF will push the $\mathrm{K}$-shell $\mathrm{X}$-ray emission to $35 \mathrm{keV}$ and beyond. Instead of standard, reflection-type crystal spectrometers, transmission-type spectrometers will be needed to measure such hard $X$ rays with high resolving power. The first-ever such measurement has been successfully accomplished under this LDRD for xenon at an X-ray energy of $31 \mathrm{keV}$ paving the way for similar measurements on NIF. 


\section{Ultra high-resolution soft x-ray spectroscopy}

The world standard for resolving power in the soft $\mathrm{x}$-ray regime is about 3000 to 6000 . The limits are mainly due to the ion motion that tends to smear out a given line. By tuning our ion source in novel ways, we produced ions which are almost motionless compared to other sources. This increased our spectroscopic resolving power to 18,800. To make use of such cold ions, we constructed a very high-resolution $(64,000)$ x-ray spectrometer.

Using this method we were able to resolve the splitting of the $2 s-2 p$ x-ray line in lithiumlike $\mathrm{Bi}^{80+}$ caused by the hyperfine interaction. The measurement also provided for the first time a test of electron-correlation contributions to the hyperfine splitting in a highly charged ion. Moreover, the measurement provided the most precise value of the Lamb shift in a highly charged ion to date. The Lamb shift was determined with an accuracy of $910^{-4}$, which is starting to approach the $610^{-6}$ measurement accuracy achieved for the neutral hydrogen atom. For the first time, it was possible to show that QED calculations are incomplete. Discrepancies with state-of-the-art calculations ranging from two to six standard errors were found, as illustrated in Fig. 2.

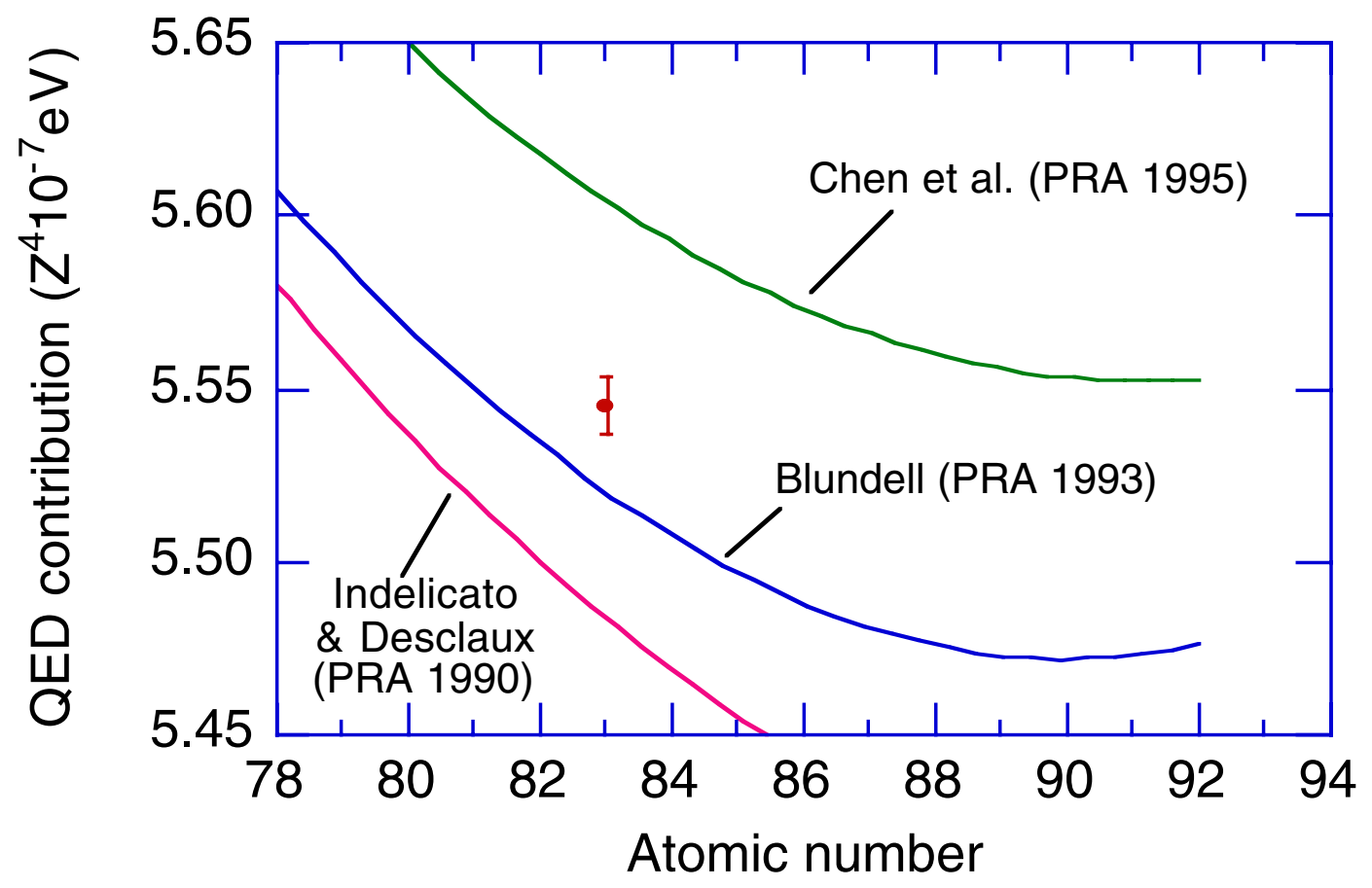

Fig. 2. Comparison of the measured and calculated QED contribution to the $2 s-2 p$ transition in highly charged Li-like ions. Solid circle and error bar indicates the result obtained with the ultra-high resolution spectrometer. 
Measurements of Ultrafast Radiative Lifetimes

Measurements of radiative transition probabilities are in their infancy compared to energy level determinations. Such measurements, however, are crucial for the development of accurate atomic calculations as they test theory in ways that energy-level measurements cannot provide, and they yield information on oscillator and absorption strengths important for line excitation processes, density diagnostics, radiation transfer, and opacity effects. Despite this glaring need only a limited number of radiative lifetimes have been measured world-wide and only in a limited range of lifetimes. Moreover, while energy levels have been measured with accuracies of up to a few parts per million, standard lifetime measurements are only accurate to within about 10 percent.

Using the ultra-high-resolution spectrometer developed above, we were able to make measurements of the line shape of the lines used as spectral diagnostics in laser fusion. The technique was demonstrated on the most prominent L-shell X-ray transition in neonlike cesium. As shown in Fig. 3, the observed line shape is Lorentzian as determined by the natural lifetime of the upper level. Using the uncertainty principle, we inferred the upper level lifetime of the line from the width of the observed line. The value we got was in the femtosecond regime (1.65 fs). This is the fastest radiative lifetime of a transition in a highly charged ion ever measured in the world.

Measurements of Long-lived Radiative Lifetimes

We developed the techniques to record the temporal evolution of the spectral lines after excitation processes are stopped and thus to complement energy level measurements with measurements of radiative lifetimes. Such measurements were performed of metastable levels of highly charged ions in the second to microsecond regime. This regime is not accessible by any existing method, such as the beam-foil technique, storage rings, or laser excitation. Our effort succeeded not only to provide lifetime measurements in regimes inaccessible with existing techniques, but also with a much improved accuracy, i.e., with an accuracy below the one-percent level. These measurements provided ultra sensitive tests of relativity and weak configuration-interaction effects on the calculated atomic wave functions.

Our effort to implement novel methods to determine accurate radiative transition probabilities has propelled us to the forefront of this area of research. We are now the record holders in the accuracy with which radiative rates of metastable levels with lifetime less than $1 \mathrm{~ms}$ can be measured. This adds to the unique capabilities we developed that makes LLNL the only laboratory in the world capable of measuring radiative lifetime in the femtosecond regime. 


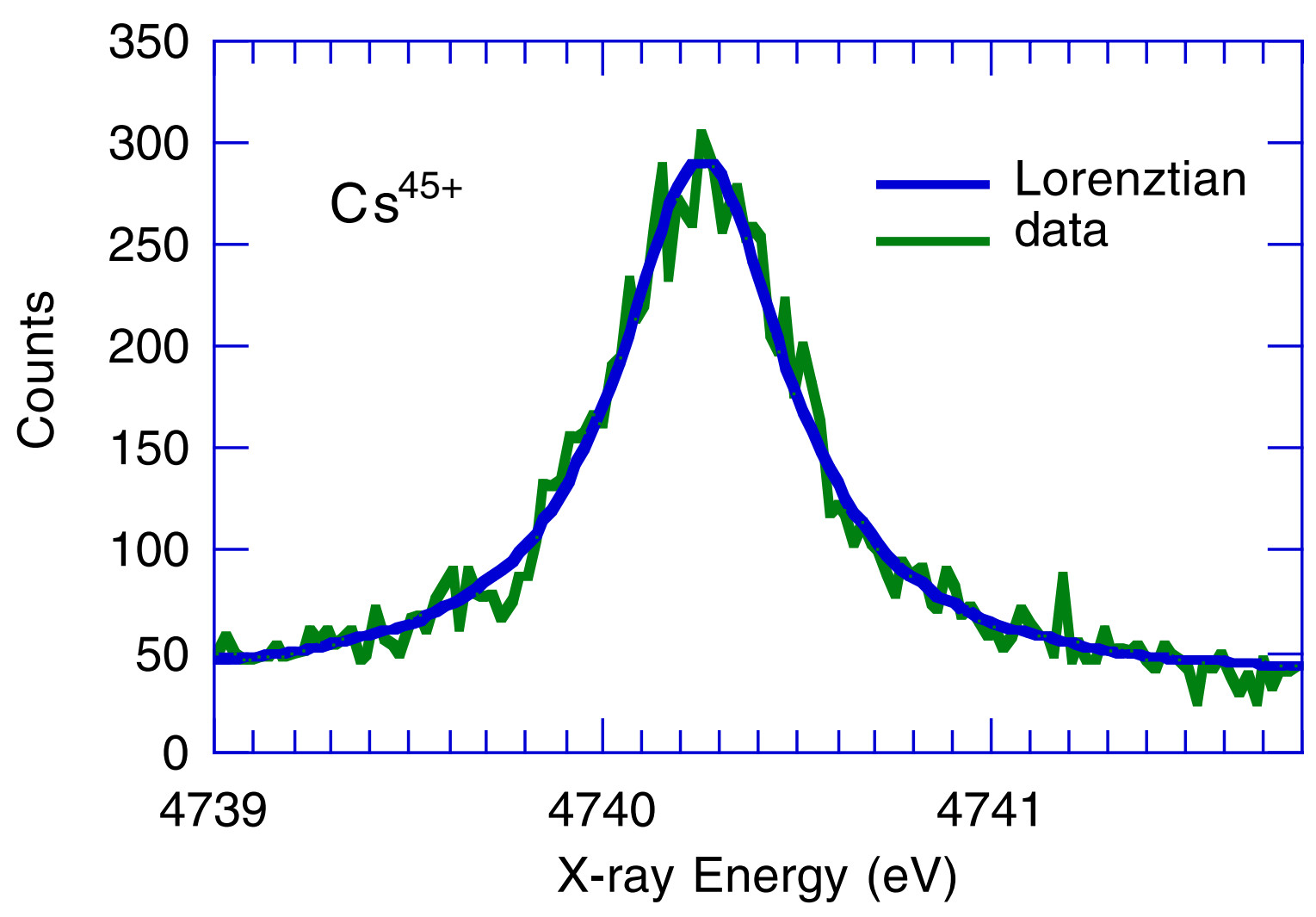

Fig. 3. Measured line width of the $2 p-3 d$ resonance transition in neonlike $\mathrm{Cs}^{45+}$ measured with the ultra-high resolution spectrometer. A 1.65-fs radiative lifetime of the upper level of the transition can be inferred from the Lorenztian line shape. The Lorenztian line shape becomes apparent only if the ions are very cold $(<100 \mathrm{eV})$ compared to the electron energy $(>4740$ $\mathrm{eV})$ needed to excite the line. 


\section{EUV Spectroscopy}

The EUV and soft x-ray region from 15-400 Å contains radiation from many charge states of such elements as $\mathrm{W}$ and $\mathrm{Au}$ important to fusion and ICF. We have opened this wavelength band to scientific scrutiny by implementing two soft x-ray spectrometers that can now produce accurate spectra on demand. To illustrate our new capabilities we reexamined spectral measurements performed on NOVA. Our new data shown in Fig. 4 has three times the accuracy and reveals much better agreement with theory than thought before.

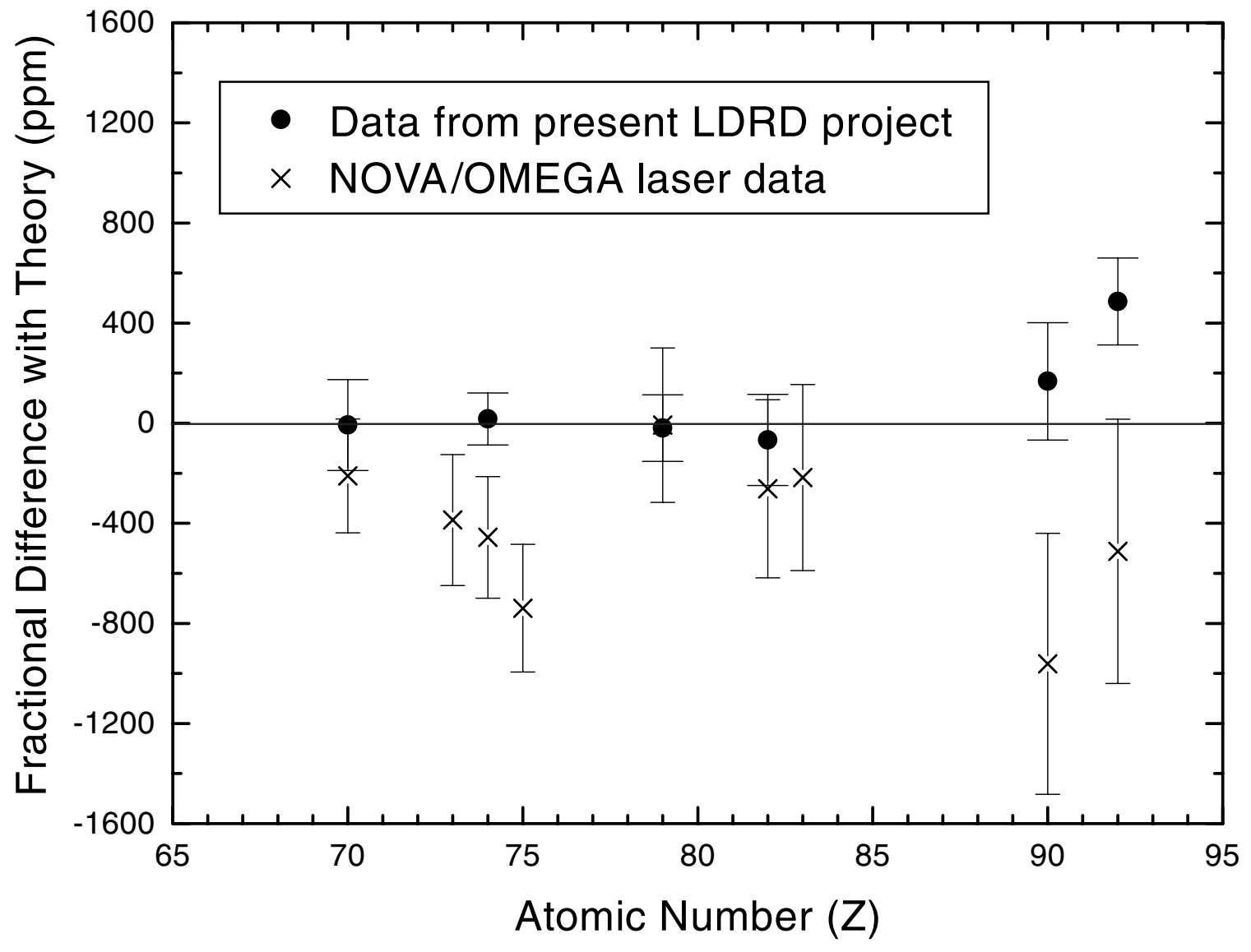

Fig. 4. Reexamination of NOVA laser measurements of the wavelengths of $4 s-4 p$ transitions in copperlike ions in the extreme ultraviolet. The data produced with our new spectrometers provide three times higher accuracy and better agreement with modern theory. 


\section{Polarization Spectroscopy}

Developing quantitative techniques for determining the magnetic sublevel populations of excited levels added another dimension to our state-of-the-art spectroscopic repertoire developed during the three years of this LDRD project. Uneven sublevel populations result in polarized lines, and we developed new instrumentation to measure polarization phenomena generated by collisions with directional electrons. The fact that the Livermore EBIT facilities employ an electron beam for exciting the ions in a well-defined geometry makes the facilities ideal for studying line polarization phenomena. Our measurements showed that polarization phenomena can be exploited to provide critical spectral signatures for detecting the presence of a directional electron beam component in plasmas, such as that generated by ultra-short laser pulses. Our measurements tested the details of electron-ion excitation calculations needed to predict polarization effects on spectral diagnostics. They also created a starting point for the design of novel polarization-based diagnostics for highdensity, laser-generated plasmas.

\section{Absolute Wavelength Standards}

Two monolithic crystals manufactured from a large silicon crystal block were successfully tested to yield new standards in the x-ray region. Each monolith has two reflecting surfaces that produce a double image of the spectral lines of interest, as shown in the figure. The spacing of the crystalline layers was absolutely calibrated in a comparison with laser frequency standards by our collaborators at the University of Jena, Germany. From the spacing of the double image and the distance between the front and back reflecting surface, absolute $\mathrm{x}$-ray wavelengths were determined for the Lyman-alpha transitions of hydrogenic $\mathrm{Si}^{13+}$ and $\mathrm{Ar}^{1 \gamma_{+}}$, proving that new $\mathrm{X}$-ray standards can be developed this way.

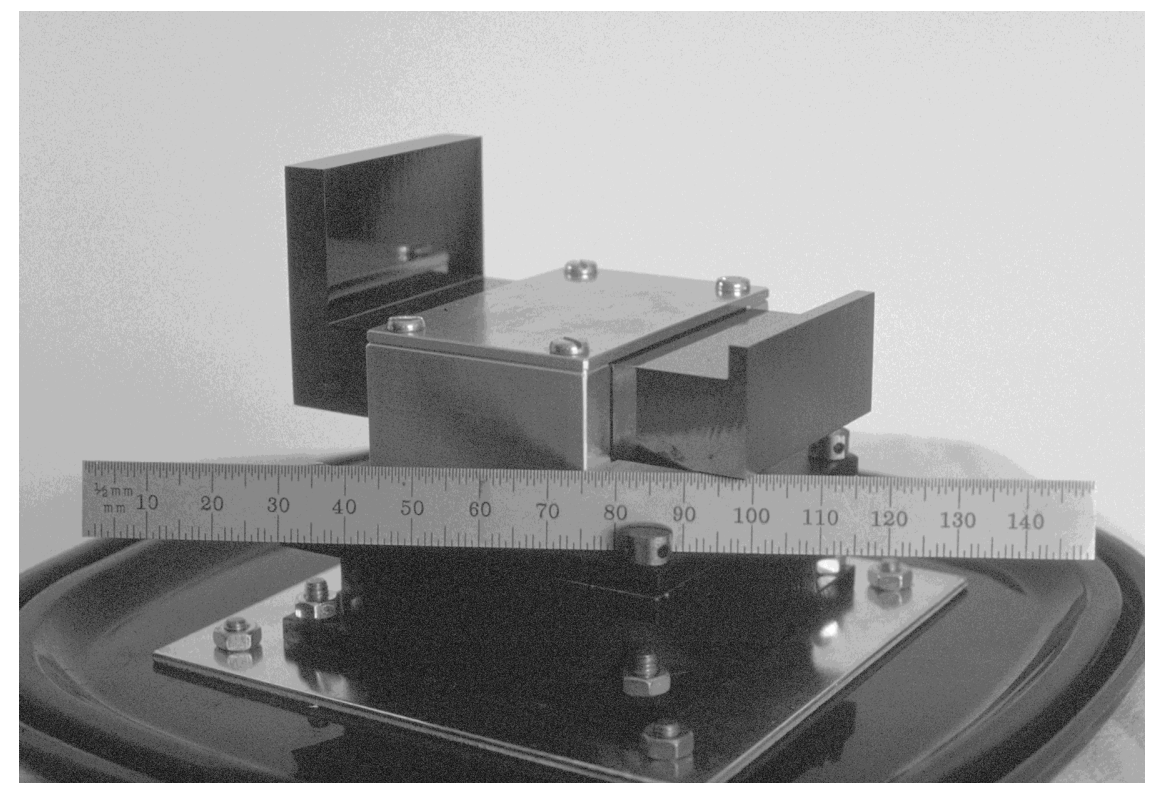

Fig. 5. Si(220) monolith used in setting new x-ray standards and testing quantum electrodynamical theory in hydrogenic ions. Two parallel reflecting surfaces were cut out of single silicon crystal and absolutely calibrated by comparison to optical frequency standards. 


\section{Conclusion:}

The results of our effort have clearly strengthen LLNL's core competency in the atomic science by creating new enabling technologies that allow unprecedented experimental access to the physics of highly charged ions. The new experimental capabilities are crucial for fully exploiting the highly charged ion capabilities unique to Livermore and for maintaining the Laboratory's lead in atomic physics research, which is a characteristic that distinguishes LLNL from any other DOE laboratory. The results obtained provide the basis for novel atomic diagnostics for high-energy-density plasmas in ICF and weapons physics relevant to science-based stockpile stewardship and for testing atomic theory models underlying many programmatic efforts. Moreover, because of their uniqueness, our results have become a stimulus to atomic theory at LLNL and world wide.

\section{Acknowledgment:}

This work was performed under the auspices of the U.S. Department of Energy by University of California Lawrence Livermore National Laboratory under contract No. W7405-Eng-48.

\section{Publications supported by this LDRD Project:}

\section{Ph. D. Thesis:}

"High-Resolution Spectroscopic Diagnostics of Very High-Temperature Plasmas in the Hard XRay Regime"

K. Widmann, 1998.

\section{Journal Publications:}

Improved electron-beam ion-trap lifetime measurement of the $1 \mathrm{~s} 2 \mathrm{~s}^{3} \mathrm{~S}_{1}$ level in $\mathrm{N}^{5+}$ and $\mathrm{F}^{7+}$ P. Neill, P. Beiersdorfer, G.V. Brown, C. Harris, E. Träbert, S. B. Utter, K.L. Wong Submitted to Physica Scripta (September 9, 1999)

Polarization of K-shell X-ray Transitions of $\mathrm{Ti}^{19+}$ and $\mathrm{Ti}^{20+}$ excited by an Electron Beam P. Beiersdorfer, G. V. Brown, S. B. Utter, P. Neill, K. J. Reed, A. J. Smith, and R. S. Thoe Physical Review A 60, 4156-4159 (1999). 
Improved Lifetime Measurement of the $\mathrm{Ne}^{8+} 1 \mathrm{~s} 2 \mathrm{~s}{ }^{3} \mathrm{~S}_{1}$ Level

E. Träbert, P. Beiersdorfer, G. V. Brown, A. J. Smith, S. B. Utter, M. F. Gu, and D. W. Savin

Physical Review A 0ㅜ, 2034-2038 (1999).

Spectroscopy in the Extreme Ultraviolet on an Electron Beam Ion Trap

P. Beiersdorfer, J. R. Crespo López-Urrutia, P. Springer, S. B. Utter, and K. L. Wong

Review of Scientific Instruments 70, 276-279 (1999).

Linear X-Ray Line Polarization Effects on Spectral Measurements Using an Electron Beam Ion Trap

P. Beiersdorfer

Proceedings of the US-Japan Workshop on Plasma Polarization Spectroscopy, Kyoto, Japan, Jan. 26 - 29, 1998, NIFS-Proceedings Series No. 37, ed. by T. Fujimoto and P. Beiersdorfer (National Institute for Fusion Studies, Gifu-ken, Japan, 1998), p. 67-89.

Forbidden Transitions in the Visible Spectra of an Electron Beam Ion Trap

E. Träbert, P. Beiersdorfer, S. B. Utter, and J. R. Crespo López-Urrutia

Physica Scripta 58, 599-604 (1998).

QED and Hyperfine Splitting in the $2 \mathrm{~s}_{1 / 2}-2 \mathrm{p}_{3 / 2}$ X-Ray Transition in Lithiumlike Bi ${ }^{80+}$

P. Beiersdorfer, K. Widmann, and J. R. Crespo López-Urrutia

Hyperfine Interactions 114, 141-146 (1998).

Precision Measurement of the Lifetime of the $1 \mathrm{~s} 2 \mathrm{~s}{ }^{3} \mathrm{~S}_{1}$ Metastable Level in Heliumlike $\mathrm{O}^{6+}$ J. R. Crespo López-Urrutia, P. Beiersdorfer, D. W. Savin, and K. Widmann

Physical Review A 모, 238-241 (1998).

Polarization-Dependent Spectra of Dielectronic Satellite Lines of Be-like Fe

A. S. Shlyaptseva, R. C. Mancini, P. Neill, P. Beiersdorfer, J. R. Crespo López-Urrutia, and K. Widmann

Physical Review A 57, 888-898 (1998).

Spectroscopy at the High-Energy Electron Beam Ion Trap (SuperEBIT)

K. Widmann, P. Beiersdorfer, J. R. Crespo López-Urrutia, and S. Elliott

In Atomic Physics with Stored Highly Charged Ions II, ed. by R. Schuch, E. Lindroth, H.-J.

Kluge (Balzer, Amsterdam, 1997), p. 73-86.

Polarization of X-Ray Li- and Be-Like Fe Satellite Lines Excited by an Electron Beam

A. S. Shlyaptseva, R. C. Mancini, P. Neill, and P. Beiersdorfer,

Rev. of Scientific Instruments $\underline{68}, 1095-1098$ (1997).

Absolute Wavelength Measurement of the Lyman- $\alpha$ Transitions of Hydrogenic Mg11+

G. Hölzer, E. Förster, D. Klöpfel, P. Beiersdorfer, G. V. Brown, J. R. Crespo López-Urrutia, and

K. Widmann

Physical Review A 구, 945-948 (1998).

Measurement of QED and Hyperfine Splitting in the $2 \mathrm{~s}_{1 / 2}-2 \mathrm{p}_{3 / 2} \mathrm{X}$-Ray Transition in ${ }^{209} \mathrm{Bi}^{80+}$ P. Beiersdorfer, A. L. Osterheld, J. Scofield, J. R. Crespo López-Urrutia, and K. Widmann Physical Review Letters $\underline{80}$, 3022-3025 (1998). 
A Quartz Quasimonolith for Absolute X-Ray Wavelength Measurements

D. Klöpfel, G. Hölzer, E. Förster, and P. Beiersdorfer

Rev. of Scientific Instruments $\underline{68}, 3669-3675$ (1997).

Polarization Spectroscopy of X-Ray Transitions from Beam-Excited Highly Charged Ions

P. Beiersdorfer, J. Crespo López-Urrutia, V. Decaux, K. Widmann, and P. Neill

Rev. of Scientific Instruments 68, 1073-1076 (1997).

High-Resolution X-Ray Spectra from Low-Temperature, Highly Charged Ions

P. Beiersdorfer

In Proceedings of the 17th International conference on X-Ray and Innershell Processes, AIP Conf. Proc. No. 389, ed. by R. L. Johnson, H. Schmidt-Böcking, and B. F. Sonntag, (American Institute of Physics, New York, 1997), p. 121-135.

Observation of Lifetime-Limited X-Ray Line Widths in Cold Highly Charged Ions P. Beiersdorfer, A. L. Osterheld, V. Decaux, and K. Widmann

Physical Review Letters 77, 5353-5356 (1996). 